\title{
EGFR gene-mutation status correlated with therapeutic decision making in lung adenocarcinoma
}

This article was published in the following Dove Press journal:

OncoTargets and Therapy

20 October 2015

Number of times this article has been viewed

\author{
Yaoyao Ren \\ Yibing Yao \\ Qing Ma \\ Diansheng Zhong \\ Oncology Department, Tianjin Medical \\ University General Hospital, Tianjin, \\ People's Republic of China
}

Correspondence: Yaoyao Ren Oncology Department, Tianjin Medical University General Hospital, 154 Anshan Road, Heping, Tianjin 300052, People's Republic of China Email prettyrenyaoyao@sina.com

\begin{abstract}
The purpose of this study was to investigate the correlation between $E G F R$-mutation status and treatment efficacy for advanced lung adenocarcinoma patients. A total of 47 patients receiving erlotinib as first-line therapy were divided into two groups: the EGFR gene mutation group included 19 patients with known EGFR-sensitive mutations, and the EGFR-mutation status-unknown group comprised 28 patients with unknown EGFR-mutation status. Both objective response rate and disease-control rate were significantly higher in the $E G F R$-mutation group compared with the $E G F R$-unknown group ( $42.1 \%$ vs $14.2 \%, P=0.032 ; 94.7 \%$ vs $57.1 \%$, $P=0.005)$. Age, sex, smoking history, stage of disease, and tissue-sample source were not significantly correlated with the distributions of mutation status. In conclusion, it is important for advanced lung adenocarcinoma patients to undergo gene analysis before being assigned a molecularly targeted drug as first-line treatment.
\end{abstract}

Keywords: $E G F R$, gene mutation, lung adenocarcinoma

\section{Introduction}

Lung cancer is one of the most common cancers and the leading cause of cancer death worldwide. The 5-year survival rate is only $16.8 \%{ }^{1-3}$ Approximately $85 \%$ of lung cancer is non-small-cell lung cancer (NSCLC), and adenocarcinoma is the most common histological type. ${ }^{1}$ Due to the lack of perceivable symptoms at the early stage, the majority of lung adenocarcinoma patients are diagnosed at advanced stages. Therefore, for a long time in the past, traditional chemotherapy was the main treatment option for these patients. However, traditional chemotherapy has a poor prognosis, because of adverse effects. Therefore, it is urgent to develop targeted therapy with more specificity.

More and more evidence has indicated that EGFR plays an important role in NSCLC, especially in adenocarcinoma. EGFR is a membrane-bound tyrosine-kinase receptor that mediates growth and survival signals. Tyrosine-kinase inhibitors (TKIs) can target the EGFR tyrosine-kinase subunit and block its function. ${ }^{4}$ Erlotinib is an oral low-molecular-weight agent. Almost $60 \%$ of erlotinib can be absorbed after oral administration. The protein-bound rate is $93 \%$. The results from the BR21 and INTEREST clinical trials recommended EGFR TKIs for second- or third-line treatment of advanced NSCLC. Meta-analysis demonstrated that erlotinib had more effectiveness for East Asian patients. Compared with traditional chemotherapy, it has better tolerability and less toxicity. ${ }^{5}$ EGFR-sensitive mutations have been proved to be strongly predictive of response to EGFR TKIs in NSCLC. ${ }^{6,7}$

Recently, a number of research results have shown that the effects of first-line TKI treatment are obviously better than traditional platinum-based double-agent chemotherapy 
in EGFR-sensitive mutation patients..$^{8-11}$ Surprisingly, newly published molecular epidemiology research of Asian advanced lung adenocarcinoma patients (PIONEER) demonstrated that EGFR-sensitive mutation rates in mainland People's Republic of China (PRC) subgroups were 50.2\%. ${ }^{12}$ Therefore, detecting such mutations could lead to the identification of subgroups of patients who would benefit substantially from TKIs. In this study, we expected to provide strong clinical research support for understanding of the molecular correlation between EGFR-mutation status and treatment efficacy for advanced lung adenocarcinoma patients.

\section{Patients and methods}

Eligibility

Patients were required to have histological documentation of primary lung adenocarcinoma, stage IIIB or stage IV, and measurable disease, as defined by RECIST (Response Evaluation Criteria In Solid Tumors). Prior chemotherapy and EGFR TKI treatment were not allowed. Previous surgery and irradiation had to have been completed at least 3 weeks before enrollment. Other eligibility criteria were age $\geq 18$ years and acceptable hepatic, renal, and hematologic function. The institutional review board of the TianJin Medical University General Hospital approved this study, and informed consent was obtained from all the participants.

\section{Populations and samples}

A total of 128 patients with pathology-proven advanced lung adenocarcinoma were enrolled from January 2010 through June 2014 at the Oncology Department of Tianjin Medical University General Hospital. Paraffin-embedded primary and metastatic tumor specimens (surgical, bronchoscopic, or computed tomography-guided needle-biopsy specimens) were collected to perform histological and gene detection. Only 56 patients' samples were available for detection of EGFR-gene mutation; the other 72 patients' EGFR-mutation status was unknown, because they refused gene detection for economic reasons.

\section{EGFR-mutation detection}

EGFR-gene mutations were detected at the SurExam Testing Center using the xTag liquid-chip method. All positive findings were independently verified by sequencing.

\section{Treatment and evaluation}

Patients received erlotinib $150 \mathrm{mg}$ orally daily on a continuous basis. One cycle of therapy was defined as 30 days. Treatment was continued until disease progression or unacceptable toxicity. The objective treatment response was assessed according to RECIST criteria prior to every third cycle of treatment.

\section{Statistical analysis}

SPSS 13.0 was used to determine the statistical significance of the mean values. A $P$-value $<0.05$ was considered to indicate a statistically significant difference.

\section{Results}

\section{EGFR-mutation status}

A total of 56 patients' specimens were available on which to perform mutation detection; 19 patients (33.9\%) had EGFRsensitive mutations (13 had deletion mutation in exon 19, and six had an L858R mutation in exon 21), and the other 37 patients had wild-type mutations.

\section{Association between EGFR mutation and clinical characteristics}

The correlation between EGFR-mutation status and clinical characteristics was analyzed. There were no significant differences in the distribution of mutation status by age, sex, smoking history, stage of disease, or tissue-sample source (primary tumor vs metastasis). Data are shown in Table 1.

\section{EGFR TKI-treatment response}

\section{Patient characteristics}

Data collection was terminated in December 2014. Nineteen patients with EGFR-sensitive mutations and 28 patients with unknown $E G F R$-mutation status agreed to take erlotinib as first-line treatment. The clinical characteristics of these patients are displayed in Table 2.

Table I Association between EGFR mutation and clinical characteristics

\begin{tabular}{lll}
\hline Clinical characteristics & EGFR mutation & $P$-value \\
\hline $\begin{array}{l}\text { Age, years } \\
\quad<60\end{array}$ & $5 / 16$ & 0.789 \\
$\quad \geq 60$ & $14 / 40$ & \\
Sex & & \\
$\quad$ Female & $14 / 36$ & 0.154 \\
$\quad$ Male & $5 / 20$ & \\
Smoking status & & \\
$\quad$ Former smoker & $3 / 18$ & 0.060 \\
$\quad$ Nonsmoker & $16 / 38$ & \\
Staging & & \\
$\quad$ IIIB & $8 / 14$ & 0.655 \\
$\quad$ IV & $11 / 42$ & \\
Specimen source & & \\
Primary tumor & $17 / 41$ & 0.219 \\
Metastasis & $2 / 15$ & \\
\hline
\end{tabular}

Note: EGFR mutation data shown as number of patients with that clinical characteristic who have EGFR mutation/total number of patients for that clinical characteristic. 
Table 2 Patient characteristics in erlotinib treatment

\begin{tabular}{lll}
\hline Clinical characteristics & EGFR-M & EGFR-UK \\
\hline Age, years & & \\
$\quad<60$ & 5 & 8 \\
$\geq 60$ & 14 & 20 \\
Sex & & \\
$\quad$ Female & 14 & 21 \\
$\quad$ Male & 5 & 7 \\
Smoking status & & \\
$\quad$ Former smokers & 3 & 4 \\
$\quad$ Nonsmokers & 16 & 24 \\
Stage & & \\
$\quad$ IIIB & 8 & 11 \\
IV & 11 & 17 \\
\hline
\end{tabular}

Abbreviations: EGFR-M, EGFR mutation; EGFR-UK, EGFR-mutation status unknown.

\section{Treatment response}

Among 19 patients who had EGFR-gene mutation, none of 19 had complete response, eight of 19 (42.1\%) had partial response, ten of $19(52.6 \%)$ had stable disease, and one of 19 (5.3\%) had progressive disease. Among 28 patients whose gene status was unknown, none of 28 had complete response, four of $28(14.2 \%)$ had partial response, 12 of $28(42.9 \%)$ had stable disease, and 12 of 28 (42.9\%) had progressive disease. Both the overall response rate (ORR) and disease-control rate (DCR) were higher in the EGFRmutation group than in the $E G F R$-unknown group ( $42.1 \%$ vs $14.2 \%, P=0.032 ; 94.7 \%$ vs $57.1 \%, P=0.005)$. Details of tumor response rates are shown in Table 3.

\section{Discussion}

A recent meta-analysis showed that the overall EGFR-mutation rate in NSCLC patients from mainland PRC was $37.5 \%$. The most common mutation types were L858R in exon 21 and del in exon 19, which accounted for 38.3\% (741 of 1,935) and $37.0 \%$ (716 of 1,935) of all EGFR-mutation types, respectively. ${ }^{13}$ Zhang et al used mutant-enriched liquid-chip technology to detect EGFR-gene mutation for NSCLC patients, and reported a mutation rate of $35.6 \% .{ }^{14}$ In our study, we used the xTag liquid chip. This is a high-throughput clinical

Table 3 EGFR TKI-treatment response

\begin{tabular}{llll}
\hline Treatment response & EGFR-M, n (\%) & EGFR-UK, n (\%) & P-value \\
\hline CR & 0 & 0 & \\
PR & $8(42.1)$ & $4(14.2)$ & \\
SD & $10(52.6)$ & $12(42.9)$ & \\
PD & $1(5.3)$ & $12(42.9)$ & \\
ORR (CR + PR) & $8(42.1)$ & $4(14.2)$ & 0.032 \\
DCR (CR + PR + SD) & $18(94.7)$ & $16(57.1)$ & 0.005 \\
\hline
\end{tabular}

Abbreviations: TKI, tyrosine-kinase inhibitor; EGFR-M, EGFR mutation; EGFR-UK, EGFR-mutation status unknown; CR, complete response; $P R$, partial response; $\mathrm{SD}$, stable disease; PD, progressive disease; ORR, overall response rate; DCR, disease-control rate. testing platform, the sensitivity of which is $1 \%$ and specificity approximately $95 \%$. The results of our study showed that the overall EGFR-mutation rate was $33.9 \%$, which was similar to the published data. The distribution of EGFR mutations was six in exon 21 (31.6\%) and 13 in exon 19 (68.4\%); we did not found mutations in exons 18 or 20.

Past epidemiological research has indicated that most lung adenocarcinoma patients with EGFR-sensitive mutations had special clinical characteristics, such as being Asian, female, and nonsmokers. However, in clinical work, we should not select patients for TKI treatment according to clinical characteristics like ethnicity, sex, or smoking history. In our study, there was no significant difference in the distributions of mutation status by age, sex, smoking history, stage of disease, or tissue-sample source. Due to the limited sample size, ongoing study will enlarge the number of cases and contribute further data for confirmation. In a recent study, the authors detected 14 samples and found that there was no significant difference in the distribution of EGFR mutation or EGFR messenger RNA-expression level by sex, age, smoking status, histological type, or stage of disease, which was consistent with this study. ${ }^{15}$

The efficiency of anticancer drugs is not only related to tumor volume or survival time but is also shown by quality of life. EGFR TKIs have relatively tolerable side effects. They have great advantages in improving patients' quality of life compared with traditional platinum-based doubleagent chemotherapy. In 2006, two Phase III trials - NEJ002 and WJTOG3405 - which compared gefitinib with standard chemotherapy in first-line treatment for EGFR-mutated NSCLC, showed that progression-free survival (PFS) in the gefitinib group was significantly longer than that in the standard chemotherapy group. ${ }^{16,17}$ These studies were the first to treat advanced NSCLC patients individually, and they indicated a road to personalized therapy by EGFRmutation status. After that, numerous studies were performed to investigate TKI as first-line treatment. In the Phase II CALGB30406 study, 181 patients who were never- or light former smokers with advanced lung adenocarcinoma were randomly assigned erlotinib alone or with carboplatin and paclitaxel, and the response rate (70\% vs $9 \%)$, PFS (14.1 vs 2.6 months), and overall survival (31.3 vs 18.1 months) favored $E G F R$-mutant patients. ${ }^{18}$ In the EURTAC trial, 174 European patients who had $E G F R$ mutations and no previous chemotherapy for metastatic disease were randomized to take either erlotinib or a platinum-based doublet, and the results showed that the median PFS was significantly longer in the erlotinib group (9.7 vs 5.2 months). ${ }^{11}$ In the PRC, the OPTIMAL trial used erlotinib to confer a significant PFS 
benefit in advanced NSCLC patients with EGFR-sensitive mutations. ${ }^{10}$ Our study also showed that patients with EGFRsensitive mutations had significantly higher ORR and DCR, which is consistent with previous clinical trails.

\section{Conclusion}

Our study demonstrated that TKI-treatment efficiency was closely associated with EGFR-mutation status, and both ORR and DCR were significantly higher in the EGFR-sensitive mutation group compared with those in the EGFR-mutationunknown group. Therefore, it is important for advanced lung adenocarcinoma patients to receive gene analysis before acceptance of a molecularly targeted drug as first-line treatment. Further studies will enlarge the case numbers to confirm this standpoint.

\section{Disclosure}

The authors report no conflicts of interest in this work.

\section{References}

1. Siegel RL, Miller KD, Jemal A. Cancer statistics, 2015. CA Cancer J Clin. 2015;65(1):5-29.

2. DeSantis CE, Lin CC, Mariotto AB, et al. Cancer treatment and survivorship statistics, 2014. CA Cancer J Clin. 2014;64(4):252-271.

3. Torre LA, Bray F, Siegel RL, Ferlay J, Lortet-Tieulent J, Jemal A. Global cancer statistics, 2012. CA Cancer J Clin. 2015;65(2):87-108.

4. Laurie SA, Goss GD. Role of epidermal growth factor receptor inhibitors in epidermal growth factor receptor wild-type non-small-cell lung cancer. J Clin Oncol. 2013;31(8):1061-1069.

5. Qi WX, Shen Z, Lin F, et al. Comparison of the efficacy and safety of EFGR tyrosine kinase inhibitor monotherapy with standard secondline chemotherapy in previously treated advanced non-small-cell lung cancer: a systematic review and meta-analysis. Asian Pac J Cancer Prev. 2012;13(10):5177-5182.

6. Herbst RS, Heymach JV, Lippman SM. Lung cancer. $N$ Engl J Med. 2008;359(13):1367-1380.

7. Huang SF, Liu HP, Li LH, et al. High frequency of epidermal growth factor receptor mutations with complex patterns in non-small cell lung cancers related to gefitinib responsiveness in Taiwan. Clin Cancer Res. 2004;10(24):8195-8203.
8. Fukuoka M, Wu YL, Thongprasert S, et al. Biomarker analyses and final overall survival results from a phase III, randomized, open-label, first-line study of gefitinib versus carboplatin/paclitaxel in clinically selected patients with advanced non-small-cell lung cancer in Asia (IPASS). J Clin Oncol. 2011;29(21):2866-2874.

9. Han JY, Park K, Kim SW, et al. First-SIGNAL: first-line single-agent Iressa versus gemcitabine and cisplatin trial in never-smokers with adenocarcinoma of the lung. J Clin Oncol. 2012;30(10):1122-1128.

10. Zhou $\mathrm{C}, \mathrm{Wu} \mathrm{YL}$, Chen G, et al. Erlotinib versus chemotherapy as first-line treatment for patients with advanced EGFR mutationpositive non-small-cell lung cancer (OPTIMAL, CTONG-0802): a multicentre, open-label, randomised, phase 3 study. Lancet Oncol. 2011; 12(8):735-742.

11. Rosell R, Carcereny E, Gervais R, et al. Erlotinib versus standard chemotherapy as first-line treatment for European patients with advanced EGFR mutation-positive non-small-cell lung cancer (EURTAC): a multicentre, open-label, randomised phase 3 trial. Lancet Oncol. 2012; 13(3):239-246.

12. Shi Y, Au JS, Thongprasert S, et al. A prospective, molecular epidemiology study of EGFR mutations in Asian patients with advanced non-small-cell lung cancer of adenocarcinoma histology (PIONEER). J Thorac Oncol. 2014;9(2):154-162.

13. Wang S, Wang Z. EGFR mutations in patients with non-small cell lung cancer from mainland China and their relationships with clinicopathological features: a meta-analysis. Int J Clin Exp Med. 2014;7(8): 1967-1978.

14. Zhang L, Yang H, Zhao Y, et al. Detection of EGFR somatic mutations in non-small cell lung cancer (NSCLC) using a novel mutantenriched liquidchip (MEL) technology. Curr Drug Metab. 2012; 13(7):1007-1011.

15. Ren GJ, Zhao YY, Zhu YJ, et al. Tumor gene mutations and messenger RNA expression: correlation with clinical response to icotinib hydrochloride in non-small cell lung cancer. Chin Med J (Engl). 2011; 124(1):19-25.

16. Maemondo M, Inoue A, Kobayashi K, et al. Gefitinib or chemotherapy for non-small-cell lung cancer with mutated EGFR. $N$ Engl J Med. 2010;362(25):2380-2388.

17. Mitsudomi T, Morita S, Yatabe Y, et al. Gefitinib versus cisplatin plus docetaxel in patients with non-small-cell lung cancer harbouring mutations of the epidermal growth factor receptor (WJTOG3405): an open label, randomised phase 3 trial. Lancet Oncol. 2010;11(2):121-128.

18. Janne PA, Wang X, Socinski MA, et al. Randomized phase II trial of erlotinib alone or with carboplatin and paclitaxel in patients who were never or light former smokers with advanced lung adenocarcinoma: CALGB 30406 trial. J Clin Oncol. 2012;30(17):2063-2069.
OncoTargets and Therapy

\section{Publish your work in this journal}

OncoTargets and Therapy is an international, peer-reviewed, open access journal focusing on the pathological basis of all cancers, potential targets for therapy and treatment protocols employed to improve the management of cancer patients. The journal also focuses on the impact of management programs and new therapeutic agents and protocols on

\section{Dovepress}

patient perspectives such as quality of life, adherence and satisfaction. The manuscript management system is completely online and includes a very quick and fair peer-review system, which is all easy to use. Visit http://www.dovepress.com/testimonials.php to read real quotes from published authors. 